\title{
A little coagulation knowledge can be dangerous!
}

\author{
Bruce D. Spiess, MD
}

Published online: 14 May 2009

(C) Canadian Anesthesiologists' Society 2009

"Elevated activated partial thromboplastin time does not correlate with heparin rebound following surgery". This understated yet considerably important manuscript is featured in this issue of the Journal. ${ }^{1}$ Medical staff who care for patients following heart surgery would do well to ponder the implications of this small yet important study. The obvious message, i.e., activated partial thromboplastin time (aPTT) following cardiopulmonary bypass (CPB) does not detect heparin, is only a surface scientific conclusion, albeit very important. There are a number of deeprooted scientific and sociologic messages here that we should also consider. These deeper revelations demonstrate how we as specialists can cling to erroneous beliefs because we have acquired incomplete data and resolute dogma.

First, the direct scientific findings need to be explored. A correlate with the manuscript's major conclusion is that reflex administration of protamine (for elevated aPTT) may be incorrect. Clearly, protamine is not an innocuous drug, as its side effects are extensively discussed elsewhere. ${ }^{2,3}$ The fact that protamine is itself an anti-thrombin agent and a platelet inhibitor is little appreciated. By administering more protamine in response to an elevated aPTT, a physician team could unwittingly increase, rather than abate, bleeding. The physician team would likely blame any bleeding on the elevated aPTT and, consequently, would likely provide positive feedback for their unsubstantiated actions. Consider the possibility of that scenario. After

\footnotetext{
B. D. Spiess, MD ( $\square)$

Department of Anesthesiology and Emergency Medicine, Virginia Commonwealth University Reanimation Engineering Shock Center (VCURES), P.O. Box 980696, Richmond, VA 23298-0695, USA

e-mail: bdspiess@vcu.edu
}

reading this paper and recognizing such reflexive ordering of protamine, the reader would be left with a strong indication that the provider lacked knowledge of blood coagulation. Some sixty years after heart surgery was first attempted, academic discussions continue today regarding the correct or "best" titration of protamine to heparin. ${ }^{4}$ In all probability, we excessively "titrate-treat" with protamine. Protamine can polyvalently reverse at least 2-3 times the amount of heparin, weight-to-weight (ex vivo), yet most centres administer either $1: 1$ or $1.3: 1$ dosages in a cookbook fashion. ${ }^{4,5}$ In the accompanying paper, no specific elevation of aPTT had corresponding evidence of heparin. An aPTT greater than twice normal could well worry practitioners that free heparin is present, but this paper shows it not to be true. In Fig. 2a, there is no relationship between aPTT and anti-Xa activity (the heparin gold standard), and the highest levels of aPTT (although only a few) had no anti-Xa activity (no heparin).

However, the aPTT elevations found after CPB did have a positive correlation to an increased international normalized ratio (INR-surrogate for prothrombin time). We have yet to understand the reason for that occurrence; however, it is fact that the INR-PT is often elevated after CPB (not considered abnormal until twofold or higher). ${ }^{6}$ Following heart surgery, no patient, unless bleeding, should be treated with fresh frozen plasma (FFP) based on an INR-PT alone. The fact that both coagulation tests have parallel elevations signifies a single mechanism. The majority of coagulation abnormalities after CPB are not found with "routine tests". ${ }^{6,7}$ For example, tissue factor pathway inhibitor (TFPI) is released from endothelial cells due to unfractionated heparin usage as well as ischemia and reperfusion. ${ }^{8}$ TFPI may account for some bleeding and its destruction could lead to thrombosis, yet we cannot track TFPI with routine testing. The authors have hypothesized 
that dysfibrinogenemia might be an explanation accounting for the aPTT and INR relationship. There is a little known fact that CPB creates oxidized fibrinogen that is dysfunctional. ${ }^{9}$ Most fibrinogen assays do not distinguish between oxidized and normal fibrinogen. A minimum fibrinogen level $\left(\geq 100 \mathrm{mg} \cdot \mathrm{dl}^{-1}\right)$ and function are required for normal aPTT and INR-PT. The fact that a patient's highly abnormal aPTT (a plasma test) can be brought into normal range by adding FFP possibly suggests either a dilutional or a dysfunctional (oxidized fibrinogen) cause. Is it of any consequence that we know the precise cause?

As long as physician teams do not "mis-treat" patients based on an elevated aPTT, perhaps it is not essential to understand the actual cause. The fundamental definition of an abnormal laboratory value needs to be called into question, since the manner in which all medical professionals comprehend laboratory tests is vital. The limits of "normal" values are defined within each individual laboratory's experience based on a "normal" (non-ill) population. Implicit in laboratory specific "normal" values is a bell-shaped population variation curve established with the upper and lower limits (two standard deviations from the mean). In cardiac surgery we urgently need to define the "normal" value for aPTT and INR-PT (as well as all other routine laboratory values) after CPB. This issue has been addressed in various large research projects, but, to date, this author has not encountered any published norms for post CPB patients. The INR-PT is frequently elevated after CPB; however, is it to be expected that an INR of 1.6 is considered "normal" for the post CPB population? Perhaps we specialists are making a fundamental error when we compare postoperative coagulation data with established laboratory "norms". Also, we may be making a second "stacked up" judgment error when we prescribe therapy for a population other than the population for which "normal values" were created. Therapy could be justified if the laboratory value were a "gold standard" (not the case with aPTT) or if laboratory testing always predicted a clinical outcome no matter the population studied (again, not the case with aPTT). Perhaps an enterprising person reading this editorial will be inspired to establish CPB population postoperative specific "normal values" (intensive care unit entry, day one, day two, etc.). Coagulation data aside, it is also important to establish "normal" values for electrolytes, creatinine, and, importantly, creatine phosphokinase and troponin. The myocardial injury proteins, in particular, stimulate debate as to what is considered a clinically significant value. Also of importance is appreciating the populace and the clinical signals of injury that could, or would, indicate an adverse outcome following CPB. I suspect many postoperative laboratory tests would not exist after CPB bell-shaped curves.
Another important scientific observation buried in the paper (Table 2) is that transfusion is associated with elevated aPTT. Is intraoperative transfusion somehow a cause of both the elevated laboratory value and the bleeding? Stored blood contains elevated levels of cytokines and platelet activating factor. Cytokines stimulate a wide range of inflammatory reactions, and platelet activation feeds back into the consumption of serine proteases. Platelet activation leads to crosstalk between coagulation and inflammatory cascades. In reality, it is possible to turn the intrinsic cascade (monitored by the aPTT) on its head, to drive the reactions backwards, and to consume more proteins in the process. Patients who are transfused more bleed more.

The aPTT and INR-PT fibrinogen concentration tests and a number of other "routine" coagulation tests have been shown as having no predictive value $(\leq 50 \%)$ regarding hemorrhage after $\mathrm{CPB} .{ }^{6,10}$ This literature notes that these tests have no positive or negative predictive value either alone or in aggregate, yet they are still widely utilized. The aPTT and INR-PT tests were designed when the serine protease cascades were being discovered. They were created as ex vivo highly contrived tests to ferret out rare congenital coagulation protein abnormalities (von Willebrand's disease, Christmas disease, etc.). The intent was never to predict who would bleed after CPB. In fact, heart surgery had not yet been conceived. Since the tests were simple, inexpensive, and readily available and could be prolonged with anticoagulants, the medical profession assumed leaps of faith by ascribing clinical diagnostic capabilities to the tests that they were not designed to accomplish. We now understand that coagulation is a complex integrative process between cells that activates and inhibits proteins, flow, shear forces, etc. A contemporary view of coagulation no longer speaks in terms of intrinsic and extrinsic cascades. Protein coagulation is a highly interactive series of simultaneous events occurring at protein binding sites on the surface of activated cells (platelets, white cells, and even erythrocytes). In recent computer models of coagulation, thousands of simultaneously interactive parameters have been modelled. I suspect that even this level of complexity is contrived and Neolithic in concept relative to true biology. Why do we persist in using the aPTT and PT tests when our cardiac textbooks teach that these tests do not predict bleeding? Now we know the aPTT after CPB has no relationship whatsoever to heparin levels. Nevertheless, the introduction of this otherwise learned paper mentions Canadian physicians using the aPTT in their practices.

There are existing coagulation tests and algorithms for coagulation monitoring-intervention that both predict bleeding and provide effective treatment. ${ }^{10-12}$ The thromboelastogram $\left(\mathrm{TEG}^{\circledR}\right)$ is one test among a number of 
others, including Hemodyne ${ }^{\mathrm{TM}}$, Sonoclot ${ }^{\circledR}$, ROTEM ${ }^{\circledR}$, PFA- $100^{\circledR}$, and Platelet Works ${ }^{\circledR}$. The TEG ${ }^{\circledR}$ and ROTEM ${ }^{\circledR}$ have been shown to have an $80-95 \%$ predictive value for postoperative hemorrhage. ${ }^{10-13}$ The unstimulated TEG ${ }^{\circledR}$ is one of the most sensitive tests for trace amounts of heparin. ${ }^{14}$ Furthermore, by using a heparinase $\mathrm{TEG}^{\circledR}$ cup simultaneously with a cellite-kaolin activated cup, the difference in R-value time explicitly denotes the presence of trace heparin. ${ }^{14} \mathrm{We}$ cardiac physicians continue to complain about bleeding, yet we do not widely adopt the technologies that have been tested and reported in our literature. The effective technologies are often referred to in the literature (this paper included) as "bed side" tests or non-mainstream tests. Yet, for $\mathrm{TEG}^{\circledR}$, in particular, there are more than 2,500 references in the published literature. How much more mainstream are we hoping for than this? When the aPTT does not predict bleeding and does not predict excess heparin after surgery, and we persist in the mis-belief that bleeding is caused by heparin rebound, we need to take a serious look at ourselves and how coagulation is taught to the next generation of surgeons and anesthesiologists. We tend to perpetuate myths and misinformation from one generation of physicians to another. Systems are the problem, and it is incumbent upon us to demand adequate laboratory support to make accurate clinical decisions. Consider the fact that physicians believed in the power of lancing, cupping, and blood letting to cure disease, and they taught these methods to each new generation for hundreds of years. Hence, a little coagulation and blood knowledge can be dangerous! Let us relinquish the dogma and re-examine the science.

\section{Un petit peu de connaissances sur la coagulation peut s'avérer dangereux!}

«Un temps de céphaline activée élevé n'est pas associé à un rebond d'héparine après une chirurgie cardiaque. » $\mathrm{Ce}$ manuscrit sobre mais très important est présenté dans ce numéro du Journal. ${ }^{1}$ Le personnel médical qui prend soin des patients à la suite d'une chirurgie cardiaque devrait réfléchir aux implications de cette étude, à la fois petite et importante. Le message évident, soit que le temps de céphaline activée (aPTT) après la circulation extra-corporelle (CEC) n'est pas un indicateur de l'héparine, n'est, malgré son importance capitale, qu'une conclusion scientifique de surface. En effet, il existe dans ce manuscrit plusieurs autres messages scientifiques et sociologiques bien ancrés sur lesquels nous devrions également nous arrêter. Ces révélations plus profondes démontrent comment nous, les spécialistes, pouvons nous attacher à des croyances erronées en raison d'une acquisition de données incomplètes et de dogmes absolus.

En premier lieu, les résultats scientifiques directs doivent être explorés. Une conséquence de la conclusion principale du manuscrit est que l'administration réflexe de protamine (pour corriger un aPTT élevé) pourrait être incorrecte. Il est clair pour tous que la protamine n'est pas un médicament inoffensif, et ses effets secondaires sont discutés en profondeur ailleurs. ${ }^{2,3}$ Le fait que la protamine soit elle-même une antithrombine et un inhibiteur plaquettaire est peu reconnu. En administrant davantage de protamine en réponse à un aPTT élevé, une équipe médicale pourrait inconsciemment augmenter plutôt que réduire les saignements du patient. L'équipe médicale accuserait vraisemblablement l'aPTT élevé de tout saignement et, par conséquent, fournirait probablement une rétroaction positive pour son action injustifiée. Pensez à la possibilité d'un tel scénario. Après avoir lu cet article et reconnu qu'une telle administration de protamine n'est basée que sur un réflexe, le lecteur se retrouverait avec plusieurs indications soulignant le manque de connaissances concernant la coagulation. Quelque soixante ans après la première tentative de chirurgie cardiaque, les discussions scientifiques concernant la titration correcte ou « la meilleure » titration de protamine à l'héparine vont encore bon train. ${ }^{4} \mathrm{Il}$ est fort probable que nous utilisions trop de protamine pour « titrer - traiter ». La protamine peut inverser au moins 2 à 3 fois la quantité d'héparine à un poids égal (ex vivo); pourtant, la plupart des centres administrent des doses de 1:1 ou 1,3:1, comme s'ils suivaient une recette de cuisine. ${ }^{4,5}$ Dans l'article présenté ici, aucune élévation spécifique de l'aPTT n'a démontré de données correspondantes d'héparine. Un aPTT plus élevé que le double de la normale devrait inquiéter les praticiens quant à la présence d'héparine, mais cet article montre que c'est faux. Dans la Fig. 2a, il n'y a aucune relation entre l'aPTT et l'activité anti-Xa (l'étalon or de l'héparine), et les taux d'aPTT les plus élevés (bien que peu nombreux) ne présentaient aucune activité anti-Xa (pas d'héparine).

Les élévations d'aPTT après la CEC démontraient cependant une corrélation positive à un rapport international normalisé accru (INR - un substitut du temps de prothrombine). La raison de cette survenue nous est encore inconnue; toutefois, il est clair que l'INR-PT est souvent élevé après la CEC (il n'est pas considéré anormal avant d'être le double ou plus). ${ }^{6}$ Après une chirurgie cardiaque, il ne faudrait traiter aucun patient, sauf s'il saigne, avec du plasma frais congelé (PFC) en se fondant uniquement sur un INR-PT. Le fait que les deux tests de coagulation montrent des élévations parallèles est indicateur d'un mécanisme unique. La majorité des anomalies de coagulation après la CEC ne sont pas dépistées avec les «tests 
courants ». ${ }^{6,7}$ Par exemple, l'inhibiteur de la voie du facteur tissulaire (TFPI) est libéré des cellules endothéliales en raison de l'utilisation d'héparine non fractionnée mais aussi en raison d'un phénomène d'ischémie et reperfusion. ${ }^{8}$ Le TFPI pourrait être la cause d'une partie du saignement, et sa destruction pourrait provoquer une thrombose, mais nous ne pouvons pas détecter le TFPI avec les tests courants. Les auteurs ont émis l'hypothèse que la dysfibrinogénémie pourrait expliquer la relation entre l'aPTT et l'INR. Nous ne disposons que de très peu de données démontrant que la CEC engendre un fibrinogène oxydé qui est dysfonctionnel. ${ }^{9}$ En effet, la plupart des tests fibrinogéniques ne font pas de distinction entre un fibrinogène oxydé et un fibrinogène normal. Un niveau $\left(\geq 100 \mathrm{mg} \cdot \mathrm{dl}^{-1}\right)$ et une fonction minimaux de fibrinogène sont nécessaires pour obtenir un aPTT et un INR-PT normaux. Le fait que l'aPTT très anormal d'un patient puisse être ramené à des niveaux normaux en ajoutant du PFC pourrait suggérer soit une cause de dilution ou de dysfonction (fibrinogène oxydé). Mais faut-il absolument connaître la cause précise ?

Tant que les équipes médicales ne «mal-traitent» pas les patients sur la base d'un aPTT élevé, peut-être n'est-il pas nécessaire de comprendre la cause réelle. La définition fondamentale d'une valeur de laboratoire anormale doit être remise en question, étant donné que la façon dont les professionnels de la médecine comprennent les tests de laboratoire est cruciale. Les limites des valeurs «normales » sont définies selon l'expérience de chaque laboratoire individuel et sur la base d'une population «normale » (c'est-à-dire non malade). Ces valeurs «normales » spécifiques à chaque laboratoire impliquent une courbe de variation de la population en forme de cloche, et elles sont établies avec des limites supérieures et inférieures (deux écarts types de la moyenne). En chirurgie cardiaque, il est urgent de définir la valeur «normale » de l'aPTT et de l'INR-PT (ainsi que d'autres valeurs de laboratoire courantes) en période post-CEC. La question a été examinée dans plusieurs projets de recherche à grande échelle mais, à l'heure actuelle, nous n'avons pas encore vu de normes publiées concernant les patients post-CEC. L'INR-PT est souvent élevé après une CEC ; toutefois, devons-nous nous attendre à ce qu'un INR de 1,6 soit considéré comme « normal » dans une population post-CEC ? Il est possible que nous, les spécialistes, fassions une erreur majeure en comparant les données de coagulation postopératoire avec les «normes » de laboratoire établies. En outre, est-il possible aussi que nous fassions des erreurs de jugement « par extension » lorsque nous prescrivons des traitements pour une population autre que celle pour laquelle les « valeurs normales » ont été créées ? Le traitement pourrait être justifié si la valeur de laboratoire était un «étalon or » (ce qui n'est pas le cas de l'aPTT) ou si les tests de laboratoire prédisaient toujours un devenir clinique, indépendamment de la population à l'étude (ce qui n'est pas le cas avec l'aPTT non plus). À la lecture de cet éditorial, il est possible qu'une personne entreprenante décide d'établir des « valeurs normales » postopératoires spécifiques pour la population subissant une CEC (à l'admission aux soins intensifs, au premier jour, au deuxième jour, etc.). Hormis les données de coagulation, il est tout aussi important d'établir des valeurs « normales » pour les électrolytes, la créatinine et, tout particulièrement, la créatinine phosphokinase et la troponine. Les protéines dénotant une lésion myocardique, en particulier, suscitent le débat quant à ce qui devrait être considéré comme une valeur pertinente du point de vue clinique. Il est également important d'évaluer la population et les signaux cliniques de lésion qui pourraient indiquer un devenir néfaste après la CEC. Je suppose que nombre de tests de laboratoire postopératoires ne se feraient plus une fois la établie la distribution normale en forme de cloche après la CEC.

Une autre observation scientifique d'importance cachée dans cet article (tableau 2) est le fait que la transfusion est associée à un aPTT élevé. La transfusion peropératoire serait-elle d'une certaine façon la cause aussi bien de la valeur de laboratoire élevée que du saignement? Le sang de banque contient des niveaux élevés de cytokines et de facteur d'activation plaquettaire. Les cytokines stimulent toutes sortes de réactions inflammatoires, et l'activation plaquettaire n'est possible que grâce à la consommation de sérines protéases. L'activation plaquettaire engendre une excitation croisée entre la coagulation et les cascades inflammatoires. En réalité, il est possible d'inverser la cascade intrinsèque (surveillée par l'aPTT), d'inverser les réactions et de consommer davantage de protéines dans le même processus. Les patients qui reçoivent plus de transfusions saignent plus.

Il a été démontré que les tests de concentration fibrinogénique de l'aPTT et de l'INR-PT ainsi que plusieurs autres tests « courants » de coagulation n'ont pas de valeur prédictive $(\leq 50 \%)$ en ce qui concerne l'hémorragie après la CEC. ${ }^{6,10}$ Ces articles soulignent que ces tests n'ont pas de valeur prédictive positive ou négative, qu'ils soient réalisés seuls ou combinés à d'autres; pourtant, ils sont encore couramment utilisés. Les tests de l'aPTT et de l'INR-PT ont été conçus à l'époque où les cascades de sérines protéases étaient découvertes. Ils ont été créés en tant que tests ex vivo très formels afin de dépister des anomalies protéiniques de coagulation congénitales rares (maladie de von Willebrand, hémophilie B, etc.). L'objectif de ces tests n'a jamais été de prédire qui saignerait après la CEC. En fait, la chirurgie cardiaque n'avait pas encore vu le jour. Mais, comme ces tests étaient simples, peu coûteux et faciles à obtenir, en plus du fait qu'ils pouvaient être prolongés à l'aide d'anticoagulants, la profession médicale 
a déclaré un véritable acte de foi en attribuant à ces tests des capacités de diagnostic clinique pour lesquels ils n'avaient pas été conçus. Aujourd'hui, nous comprenons que la coagulation est un processus intégré complexe entre les cellules qui active et inhibe les protéines, le débit, les forces de cisaillement, etc. Les définitions actuelles de la coagulation ne sont plus exprimées en termes de cascades intrinsèques et extrinsèques. La coagulation protéinique est une série très interactive d'événements simultanés survenant aux sites de liaison protéinique à la surface des cellules activées (plaquettes, leucocytes, et même érythrocytes). Dans des modèles informatiques récents de la coagulation, des milliers de paramètres simultanément interactifs ont été représentés. Selon moi, même un tel niveau de complexité est artificiel et préhistorique lorsque comparé à la biologie réelle de ce phénomène. Pourquoi persistons-nous dans l'utilisation des tests de l'aPTT et du PT alors que nos manuels de cardiologie nous enseignent que ces tests ne prédisent pas le saignement ? Nous savons désormais que l'aPTT après la CEC n'est aucunement lié aux niveaux d'héparine. Pourtant, l'introduction de cet article autrement érudit mentionne que les médecins canadiens utilisent l'aPTT dans leur pratique.

Il existe des tests de coagulation et des algorithmes pour le monitorage et l'intervention en matière de coagulation qui prédisent le saignement et procurent un traitement efficace. ${ }^{10-12}$ L'un de ces tests est le thromboélastogramme $\left(\mathrm{TEG}^{\circledR}\right)$, tout comme Hemodyne ${ }^{\mathrm{TM}}$, Sonoclot ${ }^{\circledR}$, ROTEM $^{\circledR}$, PFA- $100^{\circledR}$, et Platelet Works ${ }^{\circledR}$. Il a été démontré que les tests TEG $^{\circledR}$ et ROTEM $^{\circledR}$ ont une valeur prédictive de 80 à $95 \%$ en ce qui a trait à l'hémorragie postopératoire ${ }^{10-13} \mathrm{Le}$ $\mathrm{TEG}^{\circledR}$ non stimulé est l'un des tests les plus sensibles pour détecter les traces d'héparine. ${ }^{14}$ De plus, en utilisant une cellule $\mathrm{TEG}^{\circledR}$ à l'héparinase en même temps qu'une cellule activée à la cellite-kaoline, la différence de valeurs $\mathrm{R}$ indique explicitement la présence de traces d'héparine. Alors que nous, spécialistes en chirurgie cardiaque, ne cessons de nous plaindre du saignement, nous n'adoptons toutefois pas toutes les technologies testées et présentées dans la littérature qui nous sont adressées. Les technologies efficaces sont souvent présentées dans la littérature (notamment dans cet article) comme des tests « au chevet du patient » ou peu usités. Pourtant, pour le TEG $^{\circledR}$ tout particulièrement, il existe plus de 2500 références publiées dans la littérature. En faut-il encore plus pour que nous considérions ce test comme courant? Lorsque l'aPTT ne prédit pas le saignement et ne prédit pas l'excès d'héparine après une chirurgie, et que nous persistons dans notre fausse croyance qui veut que le saignement soit causé par un rebond d'héparine, il nous faut sérieusement nous remettre en question, tout comme il faut que nous remettions en question la façon dont la coagulation est enseignée à la prochaine génération de chirurgiens et d'anesthésiologistes.
Nous avons tendance à perpétuer les mythes et la désinformation d'une génération de médecins à la suivante. Les systèmes sont le problème, et il nous incombe d'exiger un soutien adapté de la part des laboratoires afin de pouvoir prendre des décisions cliniques précises. Gardez à l'esprit que les médecins croyaient au pouvoir des incisions, des ventouses, et des saignées pour soigner des maladies, et ils ont enseigné ces méthodes à chaque nouvelle génération de médecins pendant des siècles. Par conséquent, un peu de connaissances concernant la coagulation et le sang peut être dangereux! Abandonnons le dogme et réexaminons la science.

Conflicts of interest None declared.

\section{References}

1. Taneja $R$, Marwaha $G$, Sinha $P$, et al. Elevated activated partial thromboplastin time does not correlate with heparin rebound following surgery. Can J Anesth 2009; 56 : 7 (this issue). doi: 10.1007/s12630-009-9098-6.

2. Park $K W$. Protamine and protamine reactions. Int Anesthesiol Clin 2004; 42: 135-45.

3. Nybo M, Madsen JS. Serious anaphylactic reactions due to protamine sulfate: a systematic literature review. Basic Clin Pharmacol Toxicol 2008; 103: 192-6.

4. Levy JH, Tanaka KA. Anticoagulation and reversal paradigms: is too much of a good thing bad? Anesth Analg 2009; 108: 692-4.

5. Warkentin TE, Crowther MA. Reversing anticoagulants both old and new. Can J Anesth 2002; 49: S11-25.

6. Gravlee GP, Arora S, Lavender SW, et al. Predictive value of blood clotting tests in cardiac surgical patients. Ann Thorac Surg 1994; 58: 216-21.

7. Fassin W, Himpe D, Alexander JP, et al. Predictive value of coagulation testing in cardiopulmonary bypass surgery. Acta Anaesthesiol Belg 1991; 42: 191-8.

8. Donahue BS, Gailani D, Mast AE. Disposition of tissue factor pathway inhibitor during cardiopulmonary bypass. J Thromb Haemost 2006; 4: 1011-6.

9. Roitman EV, Azizova OA, Morozov YA, Aseicher AV. The effect of oxidized fibrinogens on blood coagulation. Bull Exp Biol Med 2004; 138: 245-7.

10. Spiess BD, Tuman KJ, McCarthy RJ, DeLaria GA, Schillo R, Ivankovich $A D$. Thromboelastography as an indicator of postcardiopulmonary bypass coagulopathies. J Clin Monit 1987; 3: 25-30.

11. Royston D, von Kier $S$. Reduced haemostatic factor transfusion using heparinase-modified thrombelastography during cardiopulmonary bypass. Br J Anaesth 2001; 86: 575-8.

12. Shore-Lesserson L, Manspeizer HE, DePerio M, Francis S, VelaCantos F, Ergin MA. Thromboelastography-guided transfusion algorithm reduces transfusions in complex cardiac surgery. Anesth Analg 1999; 88: 312-9.

13. Mengistu AM, Wolf MW, Boldt J, Röhm KD, Lang J, Piper SN. Evaluation of a new platelet function analyzer in cardiac surgery: a comparison of modified thromboelastography and whole-blood aggregometry. J Cardiothorac Vasc Anesth 2008; 22: 40-6.

14. Mittermayr M, Margreiter J, Velik-Salchner C, et al. Effects of protamine and heparin can be detected and easily differentiated by modified thromboelastography (ROTEM): an in vitro study. Br J Anaesth 2005; 95: 310-6. 